Original Research Paper

\title{
Bivariate Poisson-Lindley Distribution with Application
}

\author{
${ }^{1}$ Hossein Zamani, ${ }^{2}$ Pouya Faroughi and ${ }^{3}$ Noriszura Ismail \\ ${ }^{1}$ Department of Statistics, Hormozgan University, Bandarabbas, Iran \\ ${ }^{2}$ Department Statistics, Sanandaj Branch, Islamic Azad University, Sanandaj, Iran \\ ${ }^{3}$ School of Mathematical Sciences, Faculty of Science and Technology, \\ Universiti Kebangsaan Malaysia, 43600 UKM Bangi, Selangor, Malaysia
}

\author{
Article history \\ Received: 25-09-2014 \\ Revised: 31-12-2014 \\ Accepted: 27-01-2015 \\ Corresponding Author: \\ Noriszura Ismail \\ School of Mathematical \\ Sciences, Faculty of Science \\ and Technology Universiti \\ Kebangsaan Malaysia, \\ 43600 UKM Bangi, \\ Selangor, Malaysia \\ Email: ni@ukm.edu.my
}

\begin{abstract}
This study applies a Bivariate Poisson-Lindley (BPL) distribution for modeling dependent and over-dispersed count data. The advantage of using this form of BPL distribution is that the correlation coefficient can be positive, zero or negative, depending on the multiplicative factor parameter. Several properties such as mean, variance and correlation coefficient of the BPL distribution are discussed. A numerical example is given and the BPL distribution is compared to Bivariate Poisson (BP) and Bivariate Negative Binomial (BNB) distributions which also allow the correlation coefficient to be positive, zero or negative. The results show that BPL distribution provides the smallest Akaike Information Criterion (AIC), indicating that the distribution can be used as an alternative for fitting dependent and over-dispersed count data, with either negative or positive correlation.
\end{abstract}

Keywords: Bivariate, Poisson-Lindley, Dependent, Over-Dispersed, Count Data

\section{Introduction}

Besides mixture approach, several bivariate discrete

Mixed Poisson and mixed negative binomial distributions have been considered as alternatives for fitting count data with overdispersion. Examples of mixed Poisson and mixed negative binomial distributions are Negative Binomial (NB) obtained as a mixture of Poisson and gamma, Poisson-Lindley (PL) (Sankaran, 1970; Ghitany et al., 2008), Poisson-Inverse Gaussian (PIG) (Trembley, 1992; Willmot, 1987), Negative BinomialPareto (NBP) (Meng et al., 1999), Negative BinomialInverse Gaussian (NBIG) (Gomez-Deniz et al., 2008), negative binomial-Lindley (NBL) (Zamani and Ismail, 2010; Lord and Geedipally, 2011) and Poisson-Weighted Exponential (PWE) (Zamani et al., 2014a) distributions.

Based on literatures, the mixture approaches have been used to derive new families of bivariate distribution. The Bivariate Negative Binomial (BNB), Bivariate PoissonLognormal (BPLN), Bivariate Poisson-Inverse Gaussian (BPIG) and bivariate Poisson-Lindley (BPL) distributions are several examples of classes of mixed distribution which are extended from univariate case. For further literatures, BNB distribution was studied in Marshall and Olkin (1990) and applied in Karlis and Ntzoufras (2003), tests for overdispersion and independence in BNB model were discussed in (Jung et al., 2009; Cheon et al., 2009), BPIG distribution was derived by Stein et al. (1987), BPL distribution was proposed by Gomez-Deniz et al. (2012) and Bivariate Poisson-Weighted Exponential (BPWE) was proposed in Zamani et al. (2014b). distributions have been defined using the method of trivariate reduction (Kocherlakota and Kocherlakota, 1999; Johnson et al., 1997). The BP distribution from the trivariate reduction has been used for modeling correlated bivariate count data and several applications can be found in (Holgate, 1964; Paul and Ho, 1989). Besides BP distribution, the Bivariate Generalized Poisson (BGP) distribution from the trivariate reduction has been defined and studied in Famoye and Consul (1995), where the distribution can be used for modeling correlated and under- or overdispersed bivariate count data.

In this study, we apply the BPL distribution which was derived from the product of two PL marginals with a multiplicative factor parameter. This BPL distribution can be used for bivariate count data with positive, zero or negative correlation coefficient. The rest of this study is organized as follows. Section 2 provides the univariate version of PL distribution. Several properties of the BPL distribution, such as mean, variance and correlation coefficient, are discussed in section 3. Section 4 discusses parameter estimation for the BPL and section 5 provides several tests for testing independence. Numerical illustration is provided in section 6 , where BPL distribution is fitted to the bivariate flight aborts count data. The BPL distribution is compared to BP (Lakshminarayana et al., 1999) and BNB (Famoye, 2010) distributions which also allow positive, zero or negative correlation. 


\section{Univariate Poisson-Lindley (PL) Distribution}

The Lindley $(\theta)$ distribution has the following p.d.f. (Lindley, 1958):

$$
f(x)=\frac{\theta^{2}}{\theta+1}(1+x) e^{-\theta x}, \quad x>0, \theta>0
$$

And $m . g . f$ :

$$
M_{X}(z)=\frac{\theta^{2}}{\theta+1} \frac{\theta-z+1}{(\theta-z)^{2}}
$$

Assuming the conditional random variable $Y \mid \Lambda$ follows Poisson distribution with p.m.f:

$$
\operatorname{Pr}(Y=y \mid \lambda)=\frac{e^{-\lambda \xi}(\lambda \xi)^{y}}{y !}, \quad y=0,1,2, \ldots, \quad \lambda, \mu>0
$$

The random variable $\Lambda$ is distributed as Lindley $(\theta)$, the marginal distribution of the random variable $Y$ is distributed as $P L(\theta, \xi)$ which is:

$$
\operatorname{Pr}(Y=y)=\frac{\theta^{2} \xi^{y}}{1+\theta} \frac{\theta+\xi+y+1}{(\theta+\xi)^{y+2}}, y=0,1,2, \ldots, \theta, \xi>0
$$

\section{Bivariate Poisson-Lindley (BPL) Distribution}

By setting $\xi=1$ in (2), the p.m.f. of $P L(\theta)$ distribution which is obtained in Sankaran (1970) is:

$$
\operatorname{Pr}(Y=y)=\frac{\theta^{2}(\theta+y+2)}{(\theta+1)^{y+3}}, y=0,1,2, \ldots, \theta>0
$$

With mean:

$$
E(\mathrm{Y})=\frac{\theta+2}{\theta(\theta+1)}
$$

Variance:

$$
\operatorname{Var}(Y)=\frac{\theta^{3}+4 \theta^{2}+6 \theta+2}{\theta^{2}(\theta+1)^{2}}
$$

And $m . g . f:$

$$
\begin{aligned}
& M_{Y}(z)=M_{\Lambda}\left(e^{z}-1\right)=E\left(e^{Z Y}\right) \\
& =\frac{\theta^{2}}{1+\theta} \frac{\theta+2-e^{z}}{\left(\theta-e^{z}+1\right)^{2}}
\end{aligned}
$$

In this study, we use the BPL distribution which was derived by Gomez-Deniz et al. (2012), who used the methodology proposed by Lee (1996) and ideas suggested in Sarmanov (1966). The same approach was also used by Lakshminarayana et al. (1999) for deriving BP distribution. The joint p.m.f. of $B P\left(\lambda_{1}, \lambda_{1}, \alpha\right)$ distribution, which was derived from the product of two
Poisson marginals with a multiplicative factor parameter, is defined as (Lakshminarayana et al., 1999):

$$
\begin{aligned}
& \operatorname{Pr}\left(Y_{1}=y_{1}, Y_{2}=y_{2}\right)=e^{-\lambda_{1}-\lambda_{2}} \frac{\lambda_{1}^{y_{1}} \lambda_{2}^{y_{2}}}{y_{1} ! y_{2} !} \\
& \left\{1+\alpha\left[\left(g_{1}\left(y_{1}\right)-\bar{g}_{1}\right)\left(g_{2}\left(y_{2}\right)-\bar{g}_{2}\right)\right]\right\} \\
& y_{1}, y_{2}=0,1,2, \ldots, \quad \lambda_{1}, \lambda_{2}>0
\end{aligned}
$$

where, $g_{1}\left(y_{1}\right)$ and $g_{2}\left(y_{2}\right)$ are bounded functions in $y_{1}$ and $y_{1}$ respectively. The value of $\{$.$\} in (4) is non-negative when$ $g_{t}\left(y_{t}\right)=e^{-y_{t}}$ and $\bar{g}_{t}=E\left[g_{t}\left(Y_{t}\right)\right]=E\left(e^{-Y_{t}}\right), \quad t=1,2$.

In a similar manner, the joint p.m.f. of $B P L\left(\theta_{1}, \theta_{2}, \alpha\right)$ distribution is defined as:

$$
\begin{aligned}
& P\left(Y_{1}=y_{1}, Y_{2}=y_{2}\right)=\frac{\theta_{1}^{2}\left(y_{1}+\theta_{1}+2\right)}{\left(\theta_{1}+1\right)^{y_{1}+3}} \frac{\theta_{2}^{2}\left(y_{2}+\theta_{2}+2\right)}{\left(\theta_{2}+1\right)^{y_{2}+3}} \\
& {\left[1+\alpha\left(e^{-y_{1}}-c_{1}\right)\left(e^{-y_{2}}-c_{2}\right)\right], y_{1}, y_{2}=0,1,2, \ldots, \quad \theta_{1}, \theta_{2}>0}
\end{aligned}
$$

Where:

$$
c_{t}=E\left(e^{-Y_{t}}\right)=\frac{\theta_{t}^{2}}{1+\theta_{t}} \frac{\theta_{t}+2-e^{-1}}{\left(\theta_{t}-e^{-1}+1\right)^{2}}, t=1,2
$$

We obtain $E\left(e^{-Y_{t}}\right)$ in (6) by letting $z=-1$ in m.g.f. (3). When $\alpha=0$, random variables $Y_{1}$ and $Y_{2}$ are independent, each is distributed as a marginal $P L$. Therefore, $\alpha$ is the parameter of independence.

The first five moments of $\operatorname{BPL}\left(\theta_{1}, \theta_{2}, \alpha\right)$ distribution are:

$$
\begin{gathered}
E\left(Y_{t}\right)=\mu_{t}=\frac{\theta_{t}+2}{\theta_{t}\left(\theta_{t}+1\right)}, \quad t=1,2 \\
\operatorname{Var}\left(Y_{t}\right)=\sigma_{t}^{2}=\frac{\theta_{t}^{3}+4 \theta_{t}^{2}+6 \theta_{t}+2}{\theta_{t}^{2}\left(\theta_{t}+1\right)^{2}}, \quad t=1,2
\end{gathered}
$$

And:

$$
\operatorname{Cov}\left(Y_{1}, Y_{2}\right)=\alpha\left(c_{11}-\mu_{1} c_{1}\right)\left(c_{22}-\mu_{2} c_{2}\right)
$$

where, $c_{t t}=E\left(Y_{t} e^{-Y_{t}}\right), t=1,2$ differentiating m.g.f. in (3) with respect to $z$ and letting $z=-1$, we have $\left.\frac{\partial}{\partial z} M_{Y}(z)\right|_{z=-1}=E\left(Y e^{-Y}\right)$. Thus, $c_{t t}=\frac{\theta_{t}^{2}}{1+\theta_{t}} \frac{\theta_{t} e^{-1}-e^{-2}+3 e^{-1}}{\left(\theta_{t}-e^{-1}+1\right)^{3}}, t=1,2$.

Using the variance and covariance in (7), the correlation coefficient is: 


$$
\rho_{12}=\frac{\sigma_{12}}{\sigma_{1} \sigma_{2}}=\frac{\alpha\left(c_{11}-\mu_{1} c_{1}\right)\left(c_{22}-\mu_{2} c_{2}\right)}{\sigma_{1} \sigma_{2}}
$$

From (8), $Y_{1}$ and $Y_{2}$ are independent when $\alpha=0$ and have positive and negative correlations when $\alpha>0$ and $\alpha<0$ respectively.

\section{Parameter Estimation}

The moment estimates of $B P L\left(\theta_{1}, \theta_{2}, \alpha\right)$ distribution can be obtained by equating the mean and covariance in (7) with the sample moments $\bar{y}_{t}=\frac{\sum_{i=1}^{n} y_{i t}}{n}, t=1,2$ and $s_{12}=\frac{\sum_{i=1}^{n}\left(y_{i 1}-\bar{y}_{1}\right)\left(y_{i 2}-\bar{y}_{2}\right)}{n-1}$.

Following Sankaran (1970), the unique moment estimate of $\theta_{t} \quad$ is $\tilde{\theta}_{t}=\frac{-\left(\bar{y}_{t}-1\right)+\sqrt{\left(\bar{y}_{t}-1\right)^{2}+8 \bar{y}_{t}}}{2 \bar{y}_{t}}, \bar{y}_{t}>0, t=1,2$. The moment estimate for $\alpha$ can then be computed using $\tilde{\alpha}=s_{12}\left(\tilde{c}_{11}-\tilde{c}_{1} \bar{y}_{1}\right)^{-1}\left(\tilde{c}_{22}-\tilde{c}_{2} \bar{y}_{2}\right)^{-1}$, where $\tilde{c}_{1}, \tilde{c}_{2}, \tilde{c}_{11}$ and $\tilde{c}_{22}$ are estimated values of $c_{1}, c_{2}, c_{11}$ and $c_{22}$.

The $\log$ likelihood function for $\operatorname{BPL}\left(\theta_{1}, \theta_{2}, \alpha\right)$ distribution is:

$$
\begin{gathered}
\log L=\sum_{i=1}^{n}\left\{2 \log \theta_{1}+\log \left(y_{i 1}+\theta_{1}+2\right)-\left(y_{i 1}+3\right) \log \left(\theta_{1}+1\right)\right. \\
+2 \log \theta_{2}+\log \left(y_{i 2}+\theta_{2}+2\right)-\left(y_{i 2}+3\right) \log \left(\theta_{2}+1\right) \\
\left.+\log \left[1+\alpha\left(e^{-y_{i 1}}-c_{1}\right)\left(e^{-y_{i 2}}-c_{2}\right)\right]\right\} .
\end{gathered}
$$

The $\log$ likelihood estimates of $\operatorname{BPL}\left(\theta_{1}, \theta_{2}, \alpha\right)$ can be obtained by maximizing the log likelihood in (9). The Fisher Information matrix can be obtained using the negative expectation of the second derivatives of log likelihood.

\section{Several Tests}

As mentioned previously, when $\alpha=0$, random variables $Y_{1}$ and $Y_{1}$ are independent, each is distributed as a marginal PL. For testing independence, we can test $H_{0}: \alpha=$ 0 against $H_{1}: \alpha \neq 0$ and the test can be performed using Likelihood Ratio Test (LRT), $T=2\left(\ln L_{1}-\ln L_{0}\right)$, where $\ln L_{1}$ and $\ln L_{0}$ are the models' log likelihood under their respective hypothesis. The statistic is approximately distributed as a chi-square with one degree of freedom.

The test of independence can also be performed using Wald statistic which is, $\frac{\hat{\alpha}^{2}}{\hat{\operatorname{Var}}(\hat{\alpha})}$, where $\hat{\alpha}$ is the estimate of independence parameter and $\hat{\operatorname{Var}}(\hat{\alpha})$ is its estimated variance. The Wald statistic is approximately distributed as a chi-square with one degree of freedom. The variance of parameters for BPL distribution can be estimated using the diagonal elements of the inverse of negative Hessian matrix. The elements of Hessian matrix are the second derivatives of log likelihood.

As another alternative, we can also use a score statistic, which is further discussed in Cox and Hinkley (1979). For the score test, we need the score function, $U\left(\theta_{1}, \theta_{2}, \alpha=0\right)$ and the expected information matrix, $I\left(\theta_{1}, \theta_{2}, \alpha=0\right)$, which can be obtained from the log likelihood.

The score statistic for testing $H_{0}: \alpha=0$ against $H_{1}$ : $\alpha \neq 0$ is:

$$
S=U^{\prime}\left(\hat{\theta}_{1}, \hat{\theta}_{2}, \alpha=0\right)\left[I\left(\hat{\theta}_{1}, \hat{\theta}_{2}, \alpha=0\right)\right]^{-1} U\left(\hat{\theta}_{1}, \hat{\theta}_{2}, \alpha=0\right)
$$

Where:

$$
U\left(\hat{\theta}_{1}, \hat{\theta}_{2}, \alpha=0\right)=\left.\left(\frac{\partial \ell}{\partial \theta_{1}}, \frac{\partial \ell}{\partial \theta_{2}}, \frac{\partial \ell}{\partial \alpha}\right)\right|_{\left(\theta_{1}, \theta_{2}, 0\right)}
$$

And:

$$
\begin{aligned}
& I\left(\hat{\theta}_{1}, \hat{\theta}_{2}, \alpha=0\right) \\
& =\left(\left.\begin{array}{ccc}
-E\left(\frac{\partial^{2} \ell}{\partial \theta_{1}^{2}}\right) & -E\left(\frac{\partial^{2} \ell}{\partial \theta_{1} \partial \theta_{2}}\right) & -E\left(\frac{\partial^{2} \ell}{\partial \theta_{1} \partial \alpha}\right) \\
-E\left(\frac{\partial^{2} \ell}{\partial \theta_{2} \partial \theta_{1}}\right) & -E\left(\frac{\partial^{2} \ell}{\partial \theta_{2}^{2}}\right) & -E\left(\frac{\partial^{2} \ell}{\partial \theta_{2} \partial \alpha}\right) \\
-E\left(\frac{\partial^{2} \ell}{\partial \alpha \partial \theta_{1}}\right) & -E\left(\frac{\partial^{2} \ell}{\partial \alpha \partial \theta_{2}}\right) & -E\left(\frac{\partial^{2} \ell}{\partial \alpha^{2}}\right)
\end{array}\right|_{\left(\theta_{1}, \theta_{2}, 0\right)}\right. \\
& \text { The entries for } I\left(\theta_{1}, \theta_{2}, \alpha=0\right) \quad \text { are } \\
& \mathrm{I}_{11}=\frac{2 n}{\theta_{1}^{2}}+\sum_{i=1}^{n}\left(y_{i 1}+\theta_{1}+2\right)^{-2}-n\left(\theta_{1}+1\right)^{-2}\left(\bar{y}_{1}+3\right), \quad \mathrm{I}_{12}=\mathrm{I}_{21}=0 \text {, } \\
& \mathrm{I}_{13}=\mathrm{I}_{31}=\frac{\partial c_{1}}{\partial \theta_{1}} \sum_{i=1}^{n}\left(e^{-y_{i 2}}-c_{2}\right), \mathrm{I}_{22}=\frac{2 n}{\theta_{2}^{2}}+ \\
& \sum_{i=1}^{n}\left(y_{i 2}+\theta_{2}+2\right)^{-2}-n\left(\theta_{2}+1\right)^{-2}\left(\bar{y}_{2}+3\right) \text {, } \\
& \mathrm{I}_{23}=\mathrm{I}_{32}=\frac{\partial c_{2}}{\partial \theta_{2}} \sum_{i=1}^{n}\left(e^{-y_{i 1}}-c_{1}\right) \\
& \mathrm{I}_{33}=\sum_{i=1}^{n}\left[\left(e^{-y_{i 1}}-c_{1}\right)\left(e^{-y_{i 2}}-c_{2}\right)\right]^{2} \\
& c_{t}=\theta_{t}^{2}\left(1+\theta_{t}\right)^{-1}\left(\theta_{t}+2-e^{-1}\right) \\
& \left(\theta_{t}-e^{-1}+1\right)^{-2}, \quad t=1,2 \\
& \frac{\partial c_{t}}{\partial \theta_{t}}= \\
& {\left[3 \theta_{t}^{2}+2 \theta_{t}\left(2-e^{-1}\right)\right]\left[\left(1+\theta_{t}\right)\left(\theta_{t}-e^{-1}+1\right)\right]-} \\
& \frac{\left.3 \theta_{t}-e^{-1}+3\right)\left[\theta_{t}^{3}+\left(2-e^{-1}\right) \theta_{t}^{2}\right]}{\left(1+\theta_{t}\right)^{2}\left(\theta_{t}-e^{-1}+1\right)^{3}}, t=1,2
\end{aligned}
$$


When several models are available, one can compare the models' performance based on several likelihood measures. A regularly used measure is Akaike Information Criteria (AIC) which penalizes a model with larger number of parameters and is defined as AIC $=-2 \ln L+2 p$, where $\ln L$ denotes the fitted $\log$ likelihood and $p$ the number of parameters.

\section{Application}

Table 1 provides the flight aborts count data from 109 aircrafts, where random variables $Y_{1}$ and $Y_{2}$ respectively represent the number of flight aborts in the first and second consecutive six months of a one-year period (Mitchell and Paulson, 1981). Most observed frequencies provide $\left(y_{1}, 0\right)$ and $\left(0, y_{2}\right)$ data, indicating negative correlation between $y_{1}$ and $y_{2}$. Therefore, we fit BP (Lakshminarayana et al., 1999), BNB (Famoye, 2010) and BPL (Gomez-Deniz et al., 2012) distributions to the data since these distributions can be fitted to bivariate data with positive, zero or negative correlation.

The joint p.m.f. of $B P\left(\theta_{1}, \theta_{2}, \alpha\right)$ distribution is (Lakshminarayana et al., 1999):

$$
\begin{gathered}
\operatorname{Pr}\left(Y_{1}=y_{1}, Y_{2}=y_{2}\right)=e^{-\theta_{1}-\theta_{2}} \frac{\theta_{1}^{y_{1}} \theta_{2}^{y_{2}}}{y_{1} ! y_{2} !} \\
{\left[1+\alpha\left(e^{-y_{1}}-e^{-d \theta_{1}}\right)\left(e^{-y_{2}}-e^{-d \theta_{2}}\right)\right]} \\
y_{1}, y_{2}=0,1,2, \ldots, \quad \theta_{1}, \theta_{2}>0
\end{gathered}
$$

where, $d=1-e^{-1}$. The mean, variance and covariance are $\quad E\left(Y_{1}\right)=\operatorname{Var}\left(Y_{1}\right)=\theta_{1}, \quad E\left(Y_{2}\right)=\operatorname{Var}\left(Y_{2}\right)=\theta_{2} \quad$ and $\operatorname{Cov}\left(Y_{1}, Y_{2}\right)=\alpha \theta_{1} \theta_{2} d^{2} e^{-d\left(\theta_{1}+\theta_{2}\right)}$.

The joint p.m.f. of $B N B\left(\theta_{1}, \theta_{2}, a_{1}, a_{2}, \alpha\right)$ distribution is (Famoye, 2010):

$$
\begin{gathered}
P\left(Y_{1}=y_{1}, Y_{2}=y_{2}\right)=\left(\begin{array}{c}
a_{1}^{-1}+y_{1}-1 \\
y_{1}
\end{array}\right) \theta_{1}^{y_{1}} \\
\left(1-\theta_{1}\right)^{a_{1}^{-1}}\left(\begin{array}{c}
a_{2}^{-1}+y_{2}-1 \\
y_{2}
\end{array}\right) \theta_{2}^{y_{2}}\left(1-\theta_{2}\right)^{a_{2}^{-1}} \\
\times\left[1+\alpha\left(e^{-y_{1}}-c_{1}\right)\left(e^{-y_{2}}-c_{2}\right)\right] \\
y_{1}, y_{2}=0,1,2, \ldots, \quad \theta_{1}, \theta_{2}>0, \quad 0<a_{1}, a_{2}<1
\end{gathered}
$$

Table 1. Observed and fitted values (flight aborts count data)

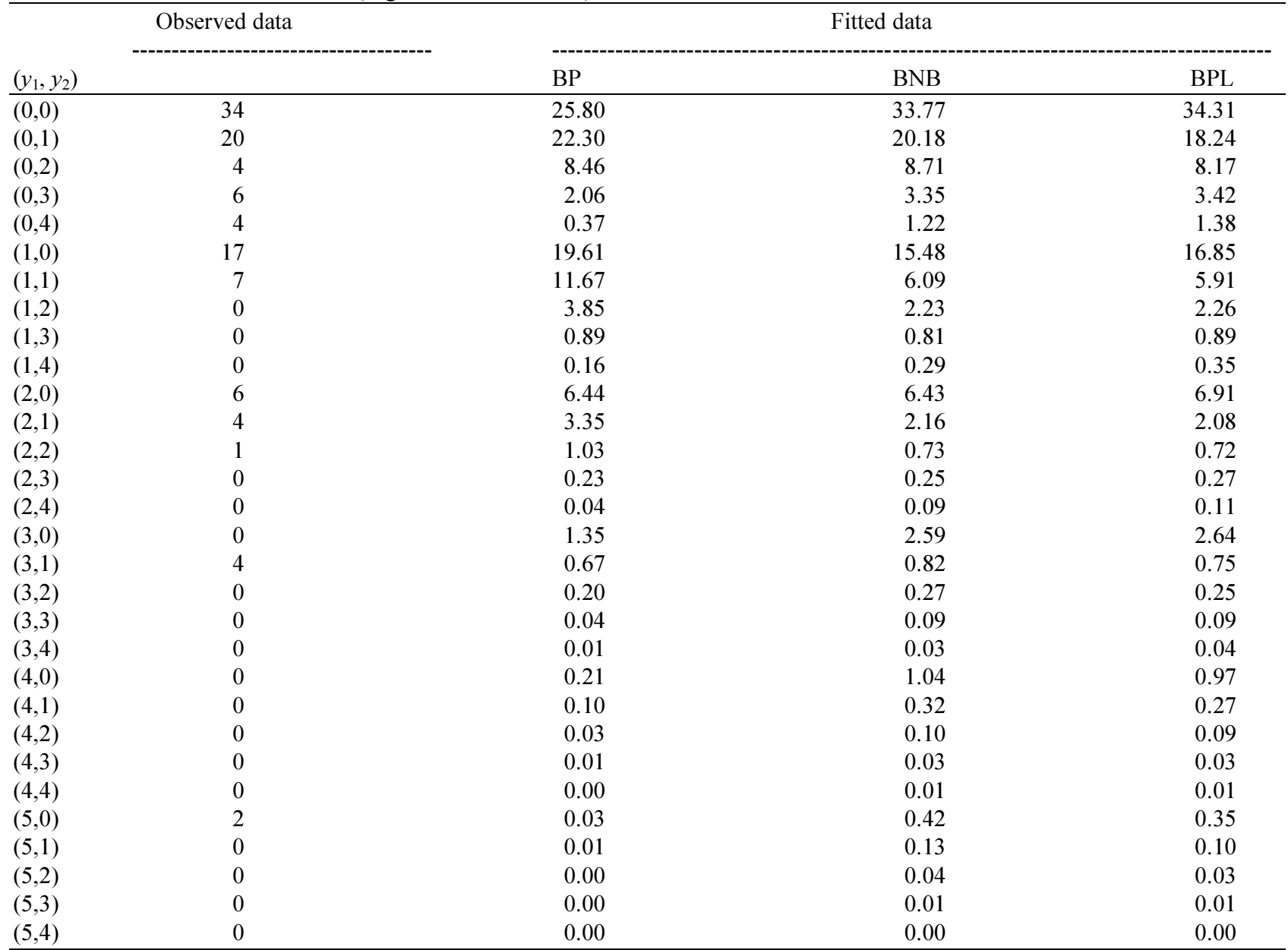


Table 2. Estimated parameters and AIC of BP, BNB and BPL distributions

\begin{tabular}{llll}
\hline Parameters & BP & BNB & BPL \\
\hline$\theta_{1}$ & 0.6129 & 0.4045 & 2.1165 \\
$\theta_{2}$ & 0.7131 & 0.3138 & 1.8607 \\
$a_{1}$ & - & 1.0977 & - \\
$a_{2}$ & - & 0.6305 & - \\
$\alpha$ & -0.9290 & -1.1109 & -1.0363 \\
$\log L$ & -254.99 & -244.27 & -244.62 \\
AIC & 515.99 & 498.54 & 495.24 \\
\hline
\end{tabular}

where, $c_{t}=E\left(e^{-Y_{t}}\right)=\left(\frac{1-\theta_{t}}{1-\theta_{t} e^{-1}}\right)^{a_{t}^{-1}}, t=1,2$ and $a_{t}, t=1,2$, is the dispersion parameter. The mean, variance and covariance are $E\left(Y_{1}\right)=a_{1}^{-1} \frac{\theta_{1}}{1-\theta_{1}}, \operatorname{Var}\left(Y_{1}\right)=a_{1}^{-1} \frac{\theta_{1}}{\left(1-\theta_{1}\right)^{2}}$, $E\left(Y_{2}\right)=a_{2}^{-1} \frac{\theta_{2}}{1-\theta_{2}}, \operatorname{Var}\left(Y_{2}\right)=a_{2}^{-1} \frac{\theta_{2}}{\left(1-\theta_{2}\right)^{2}} \quad$ and $\operatorname{Cov}\left(Y_{1}, Y_{2}\right)=\alpha c_{1} c_{2} A_{1} A_{2}$ where $A_{t}=\frac{a_{t}^{-1} \theta_{t} e^{-1}}{1-\theta_{t} e^{-1}}-\frac{a_{t}^{-1} \theta_{t}}{1-\theta_{t}}, t=1,2$. Therefore, the correlation coefficient for $B P, B N B$ and $B P L$ distributions can be positive, zero or negative, depending on the value of multiplicative factor parameter, $\alpha$. For comparison purpose, Table 1 also provides the fitted values from $B P, B N B$ and $B P L$ distributions.

Table 2 provides the estimated parameters, log likelihood and $A I C$ for $B P, B N B$ and $B P L$ distributions. It can be seen that all distributions provide negative value for $\alpha$, indicating negative correlation. Even though both BNB and BPL distributions produce similar log likelihood, the number of parameters for BPL distribution is less and thus, producing smaller $A I C$. Based on $A I C, B P L$ distribution provides the best fit for the data.

\section{Conclusion}

In this study, $B P L$ distribution has been fitted to a sample of bivariate count data. Based on the results, $B P L$ distribution provides better fit than $B P$ and $B N B$ distributions, indicating that the distribution can be used as an alternative for fitting dependent and over-dispersed count data, with either positive or negative correlation.

\section{Funding Information}

The authors gratefully acknowledge the financial support received in the form of research grants (FRGS/1/2013/SG04/UKM/02/5 and LRGS/TD/2011/UKM/ICT/03/02) from the Ministry of Higher Education (MOHE), Malaysia.

\section{Author's Contributions}

Hossein Zamani: Suggested BPL distribution and developed R program for fitting BPL distribution.

Pouya Faroughi: Developed R program for fitting $\mathrm{BP}, \mathrm{BNB}$ and BPL distributions, coordinated the data analysis and contributed to the writing of the manuscript.

Noriszura Ismail: Contributed to the writing of the manuscript, designed the research plan and carried out the overall editing of manuscript.

\section{Ethics}

This article is original and contains unpublished material. The corresponding author confirms that all of the other authors have read and approved the manuscript and no ethical issues involved.

\section{References}

Cheon, S., S.H. Song and B.C. Jung, 2009. Tests for independence in a bivariate negative binomial model. J. Korean Statist. Soc., 38: 185-190.

DOI: $10.1016 / \mathrm{j} . j \mathrm{kss} .2008 .11 .004$

Cox, D.R. and D.V. Hinkley, 1979. Theoretical Statistics. 1st Edn., CRC Press, ISBN-10: 0412161605, pp: 528.

Famoye, F. and P.C. Consul, 1995. Bivariate generalized Poisson distribution with some applications. Metrika, 42: 127-138. DOI: 10.1007/BF01894293

Famoye, F., 2010. On the bivariate negative binomial regression model. J. Applied Statist., 37: 969-981. DOI: $10.1080 / 02664760902984618$

Ghitany, M.E., B. Atieh and S. Nadarajah, 2008. Lindley distribution and its application. Math. Comput. Simulat., 78: 493-506. DOI: $10.1016 /$ j.matcom.2007.06.007

Gomez-Deniz, E., J. Maria Sarabia and N. Balakrishnan, 2012. A multivariate discrete poisson-lindley distribution: Extensions and actuarial applications. ASTIN Bull., 42: 655-678.

DOI: 10.2143/AST.42.2.2182812

Gomez-Deniz, E., J.M. Sarabia and E. Calderin-Ojeda, 2008. Univariate and multivariate versions of the negative binomial-inverse Gaussian distributions with applications. Insurance: Math. Econ., 42: 39-49.

DOI: 10.1016/j.insmatheco.2006.12.001

Holgate, P., 1964. Estimation for the bivariate Poisson distribution. Biometrika, 51: 241-245.

DOI: $10.1093 /$ biomet/51.1-2.241

Johnson, N.L., S. Kotz and N. Balakrishnan, 1997. Discrete Multivariate Distributions. 1st Edn., Wiley, New York, ISBN-10: 0471128449, pp: 328. 
Jung, B.C., M. Jhun and S.M. Han, 2009. Score test for overdispersion in the bivariate negative binomial models. J. Statist. Comput. Simulat., 79: 11-24. DOI: $10.1080 / 00949650701550606$

Karlis, D. and I. Ntzoufras, 2003. Analysis of sports data by using bivariate Poisson models. Statistician, 52: 381-393. DOI: $10.1111 / 1467-9884.00366$

Kocherlakota, S. and K. Kocherlakota, 1999. Bivariate discrete distributions. Encyclopedia Statist. Sci. DOI: 10.1002/0471667196.ess0605.pub2

Lakshminarayana, J., S.N.N. Pandit and K.S. Rao, 1999. On a bivariate Poisson distribution. Communicat. Statist. Theory Methods, 28: 267-276. DOI: 10.1080/03610929908832297

Lee, M.L.T., 1996. Properties and applications of the Sarmanov family of bivariate distributions. Communicat. Statist. Theory Methods, 25: 1207-1222. DOI: $10.1080 / 03610929608831759$

Lindley, D.V., 1958. Fiducial distributions and Bayes' theorem. J. Royal Statist. Soc., 20: 102-107.

Lord, D. and S.R. Geedipally, 2011. The negative binomialLindley distribution as a tool for analyzing crash data characterized by a large amount of zeros. Accident Anal. Prevent., 43: 1738-1742. DOI: 10.1016/j.aap.2011.04.004

Marshall, A.W. and I. Olkin, 1990. Multivariate distributions generated from mixtures of convolution and product families. Topics Statist. Depend., 16: 371-393. DOI: $10.1214 / \mathrm{lnms} / 1215457574$

Meng, S., Y. Wei and G.A. Whitmore, 1999. Accounting for individual overdispersion in a bonus-malussystem. ASTIN Bull., 29: 327-337. DOI: 10.2143/AST.29.2.504619

Mitchell, C.R. and A.S. Paulson, 1981. A new bivariate negative binomial distribution. Naval Res. Logistics Q., 28: 359-374. DOI: 10.1002/nav.3800280302
Paul, S.R. and N.I. Ho, 1989. Estimation in the bivariate poisson distribution and hypothesis testing concerning independence. Communicat. Statist. Theory Methods. 18: 1123-1133. DOI: $10.1080 / 03610928908829955$

Sankaran, M., 1970. The discrete Poisson-Lindley distribution. Biometrics, 26: 145-149. DOI: $10.1080 / 00949650701550259$

Sarmanov, O.V., 1966. Generalized normal correlation and two-dimensional Frechet classes. Doklady, Soviet Mathemat., 168: 596-599.

Stein, G., W. Zucchini and J. Juritz, 1987. Parameter estimation for the Sichel distribution and its multivariate extension. J. Am. Statist. Associat., 82: 938-944. DOI: 10.1080/01621459.1987.10478520

Trembley, L., 1992. Using the poisson inverse gaussian in bonus-malus systems. ASTIN Bulletin, 22: 97-106.

DOI: 10.2143/AST.22.1.2005129

Willmot, G.E., 1987. The Poisson-inverse Gaussian distribution as an alternative to the negative binomial. Scandinavian Actuarial J., 2: 113-127. DOI: $10.1080 / 03461238.1987 .10413823$

Zamani, H. and N. Ismail, 2010. Negative binomialLindley distribution and its application. J. Math. Statist. 6: 4-9. DOI: $10.3844 /$ jmssp.2010.4.9

Zamani, H., N. Ismail and P. Faroughi, 2014a. Poisson-weighted exponential Univariate version and regression model with applications. J. Math. Statist. 10: 148-154.

DOI: $10.3844 /$ jmssp.2014.148.154

Zamani, H., N. Ismail and P. Faroughi, 2014b. Bivariate Poisson-weighted exponential distribution with applications. AIP Proc., 1602, 964-964. DOI: $10.1063 / 1.4882600$ 